\title{
Environnement et interdisciplinarité : paradoxes dans le champ éducatif
}

La question de l'éducation relative à l'environnement a été posée de manière pressante, en France comme dans le reste du monde, depuis une trentaine d'années. Cependant, malgré rapports, proclamations, décisions, il n'est pas sûr que cette éducation ait beaucoup progressé. En effet, les prescriptions restent toujours dans le registre des injonctions ministérielles, «obligatoires », mais seulement illustrées d'exemples, selon une conception de la « diffusion de bonnes pratiques »; il y a donc brouillage sur l'importance à donner à l'éducation à l'environnement. On peut penser qu'elle est plus l'objet de préoccupations d'annonces médiatiques que de mise en place d'une réelle obligation dont les écoles seraient comptables devant la nation. Enfin, les appellations et les orientations changent très rapidement : ces toutes dernières années, $l^{\prime}$ « éducation à l'environnement » a été successivement transmuée en " éducation à l'environnement et au développement durable ", puis en "éducation au développement durable», sans d'ailleurs que les pratiques déjà très partielles à l'école ou en formation changent beaucoup. Finalement, enseignants et formateurs désireux de prendre au sérieux cette éducation à finalités civiques ne savent sans doute plus trop où en est la « doctrine». En tout cas, il serait malvenu de tenir rigueur aux enseignants des carences constatées, relativement plus importantes en France que dans d'autres pays développés, Suède ou Canada par exemple.

En réalité, lorsqu'on s'intéresse à ce qui vient d'être appelé la « doctrine », ou schéma rationnel de conception des activités scolaires, de leurs fins, contenus et modalités, on s'aperçoit que les textes qui la communiquent sont contradictoires. $L^{\prime}$ « éducation au développement durable» se heurte sans doute à des obstacles analogues à ceux que rencontrent les recherches sur et pour l'environnement, qui requièrent un minimum d'interdisciplinarité ou de multiréférentialité, incluant les sciences humaines et sociales, une prise en compte des populations et des groupes sociaux concernés avec leurs idées et leurs intérêts, une interaction avec des acteurs qui n'interviennent pas au nom de la recherche, imposant aux chercheurs une compétence transdisciplinaire. Mais cette éducation à l'environnement se heurte aussi à des obstacles qui viennent de la différence entre éducation et recherche. C'est sans doute en termes de paradoxes que ces obstacles doivent être caractérisés, paradoxes qui proviennent des différences entre actions éducatives, matières ou disciplines scolaires et disciplines académiques de formation supérieure ou de recherche.

Si l'école ou le collège sont dans l'obligation de «s'intéresser à l'environnement », c'est que les enjeux de société concernant l'environnement ne peuvent être arrêtés à leur porte : ils les concernent dans la vie scolaire elle-même, comme dans la mission socialisatrice de promouvoir une sensibilité et des dispositions à agir favorables à l'environnement et conformes à l'idée plus ou moins consensuelle que l'on se fait du rôle de l'école pour affronter ces enjeux. De ce point de vue, on peut dire qu'il y a éducation à l'environnement si et seulement si les enjeux environnementaux considérés comme majeurs dans la société, la collectivité ou la communauté concernées sont effectivement traités par leur école en tant qu'enjeux. C'est au fond ce qu'exprimait une déclaration sur l'éducation relative à l'environnement du Conseil national des programmes français, publiée en mai 1992, dont la première des deux parties avait pour titre : «Enjeux environnementaux et perspectives éducatives »; dans cet esprit, le Conseil proposait à l'époque d'aborder «dans les années qui viennent », et pour la France, sept « enjeux prioritaires », « en mettant l'accent sur l'élucidation des problèmes et l'approche de solutions ».

D'une telle conception fondatrice, découlent quatre conséquences. La première est que la visée première de l'éducation à l'environnement est de développer des dispositions (attitudes et compétences), individuelles et collectives, pour agir en faveur de l'environnement; c'est le rôle $\mathrm{d}^{\prime}$ " actions éducatives », avec réalisations collectives, 
valorisation vers la communauté, implication personnelle et débat critique. La deuxième est que l'éducation à l'environnement, prenant place à côté de disciplines du second degré visant la construction de connaissances structurées, n'est pas destinée à devenir une discipline de plus : elle doit être pensée comme un ensemble d' «actions éducatives », essentiellement non-disciplinaires dans leurs motifs. La troisième est que la question principale est celle du montage de telles actions, et non pas celle des modalités de l'interdisciplinarité de l'enseignement qui peut lui être associé pour "élucider certains problèmes ». La quatrième, enfin, est que la question de la nature des contributions des disciplines scolaires, et donc du rôle des enseignants en tant que spécialistes de ces disciplines, est ouverte : apports de connaissances qui «fondent l'action » ou mise en avant de questions pour lesquelles ces disciplines sont capables d'apporter quelques réponses validées, pouvant constituer des repères pour l'action. On comprend qu'une telle problématique de l'éducation à l'environnement ait pu inquiéter des responsables politiques et administratifs dont elle contredisait les prescriptions, même s'ils les considèrent ensuite comme inefficaces : la déclaration du Conseil national des programmes a été très rapidement oubliée. Sans en tirer toutes les conséquences, le tout récent rapport du Groupe de travail interministériel sur l'éducation au développement durable (rapport Brégeon, janvier 2008) développe cependant une problématique actualisée, mais très voisine.

Si les mots ont un sens, il demeure que l'éducation à l'environnement devrait présenter un contraste dans ses formes et ses contenus avec l'enseignement des disciplines scientifiques; contrairement à celles-ci, dont la visée à long terme est l'appropriation d'un patrimoine de connaissances, celle-là plonge l'école, les élèves, la "communauté éducative " dans le mouvement social d'élucidation, de concertation et d'action pour des enjeux sociétaux d'aujourd'hui. De ce point de vue, il n'en va sans doute pas différemment pour la recherche; mais, alors que la contribution cognitive de la recherche sur l'environnement ne peut être qu'interdisciplinaire, la contribution éducative de l'école est, au moins au départ et pour les jeunes enfants, a-disciplinaire. Dans l'incompréhension de ce point fondateur réside sans doute un des obstacles majeurs à la généralisation effective de l'éducation à l'environnement, comme d'ailleurs de la plupart des « éducations à » (la citoyenneté, la sécurité, la santé...), par opposition aux «enseignements de » (géographie, sciences physiques...) : dans leur masse, ni les administrateurs, ni les formateurs, ni bien sûr les enseignants ne sont capables de concevoir réellement des actions éducatives qui ne soient pas dans la forme de disciplines; ou plutôt, pour eux, le non-disciplinaire ne peut être que de l'interdisciplinaire. Il leur faudrait sans doute parcourir un chemin de formation professionnelle et de réflexion critique beaucoup plus long, passer de la maîtrise d'une discipline à la capacité à communiquer et coopérer dans un cadre interdisciplinaire, puis à la reconstruction transdisciplinaire qui permet d'interagir avec ceux qui n'ont aucune raison d'aborder les enjeux qui les intéressent à partir des points de vue des disciplines scientifiques. Cela ne serait sans doute pas suffisant, car les disciplines scientifiques scolaires envisagent habituellement la technique et l'action comme l'application de savoirs sûrs : en quoi est-il alors satisfait à la demande d'aborder des « enjeux environnementaux »?

Chacun a une expérience vécue de l'éducation, chacun a une opinion individuelle légitime sur l'éducation. Mais penser vraiment les problèmes de l'éducation est plus difficile qu'il n'y paraît. L'éducation à l'environnement, maintenant remplacée par l'éducation au développement durable, est sans doute une bonne illustration de la nécessité de prendre le temps de poser les problèmes, de débusquer les paradoxes, d'étudier les choix stratégiques, d'élaborer les lignes directrices.

Jean-Louis Martinand 